\title{
Finite-volume corrections to low-energy constants from the partially quenched effective theory
}

\section{Christoph Lehner* and Tilo Wettig}

Institute for Theoretical Physics, University of Regensburg, 93040 Regensburg, Germany

E-mail: christoph.lehnerephysik.uni-regensburg.de,

tilo.wettig@physik.uni-regensburg.de

We calculate finite-volume corrections to the low-energy constants $\Sigma$ and $F$ in the epsilon-regime of QCD using partially quenched chiral perturbation theory in the supersymmetry formulation without a singlet particle. We comment on how to minimize these corrections in lattice simulations of QCD.

The XXVII International Symposium on Lattice Field Theory - LAT2009

July 26-31 2009

Peking University, Beijing, China

\footnotetext{
*Speaker.
} 


\section{Introduction}

The low-energy constants (LEC) appearing in the chiral effective Lagrangian, which are of great phenomenological importance, can be determined by fitting analytical results from chiral random matrix theory (RMT) to lattice data for the eigenvalue spectrum of the Dirac operator. The lowest-order LECs are $\Sigma$ and $F$. While $\Sigma$ can be determined rather easily, e.g., from the distribution of the small Dirac eigenvalues, the extraction of $F$ is somewhat more complicated and requires the inclusion of a suitable chemical potential $[1,2]$.

Since lattice simulations are restricted to a finite volume, it is important to take into account finite-volume corrections to the RMT results, which can be obtained by going to next-to-leading order (NLO) in the $\varepsilon$-regime. Recently, finite-volume corrections to the unquenched partition function of QCD in the $\varepsilon$-regime were obtained in $[3,4]$. However, in order to extract the relevant eigenvalue correlation functions the partially quenched partition function of QCD is needed. A relatively simple method to obtain the partially quenched theory is to introduce $n$ replicated flavors in the unquenched theory and then to analytically continue in the discrete number of quark flavors to zero. This so-called replica trick was first used in the theory of disordered systems [5]. It is well known that the replica trick is potentially problematic since the analytic continuation from an isolated set of points is not uniquely defined.

In this contribution we choose to use an alternative way to obtain the partially quenched theory that does not suffer from the potential problems of the replica trick and can therefore be used to check and extend previous results. In addition to the sea quarks, we introduce fermionic and bosonic valence quarks. In nuclear physics and condensed matter physics this method is known as the supersymmetry method or Efetov method for quenched disorder [6]. In the context of QCD this idea was first used by Morel [7]. The effective low-energy theory of QCD with $N_{f}+N_{v}$ quarks and $N_{v}$ bosonic quarks was developed by Bernard and Golterman [8] and by Sharpe and Shoresh [9]. In this work we use the effective theory without a singlet particle as discussed by Sharpe and Shoresh and consider it in a finite volume and for small quark masses. In order to access $F$ in addition to $\Sigma$, we include an imaginary quark chemical potential $\mu[1,2]$. (A first exploratory lattice study of this idea was performed in Ref. [10].) We compute the partition function at next-to-leading order in the $\varepsilon$-regime and thereby obtain finite-volume corrections of order $1 / \sqrt{V}$ to the partially quenched theory that translate into finite-volume corrections to the LECs $\Sigma$ and $F$. Our results agree with previous results for the unquenched partition function [3,4]. The details of this calculation are given in a separate publication [11].

\section{The effective theory}

We define QCD with $N_{f}+N_{v}$ quarks and $N_{v}$ bosonic quarks by the partition function

$$
Z=\int d[A] e^{-S_{\mathrm{YM}}}\left[\prod_{f=1}^{N_{f}} \operatorname{det}\left(\not D+m_{f}\right)\right]\left[\prod_{i=1}^{N_{v}} \frac{\operatorname{det}\left(\not D+m_{v i}\right)}{\operatorname{det}\left(\not D+m_{v i}^{\prime}\right)}\right],
$$

where the integral is over all gauge fields $A, S_{\mathrm{YM}}$ is the Yang-Mills action, $\not D$ is the Dirac operator, $m_{1}, \ldots, m_{N_{f}}$ are the masses of the sea quarks, $m_{v 1}, \ldots, m_{v N_{v}}$ are the masses of the fermionic valence quarks, and $m_{v 1}^{\prime}, \ldots, m_{v N_{v}}^{\prime}$ are the masses of the bosonic valence quarks. By setting the mass $m_{v i}$ of 
a valence quark equal to the mass $m_{v i}^{\prime}$ of the corresponding bosonic quark, the ratio of determinants of this pair cancels and the flavor $i$ is quenched. The corresponding chiral effective theory in the partially quenched case of $N_{f}>0$ is defined by the Nambu-Goldstone (NG) manifold given by [9]

$$
U(x)=\exp \left[\frac{i \sqrt{2}}{F} \xi(x)\right]
$$

with

$$
\xi(x)=\left(\begin{array}{ll}
\pi(x) & \bar{\kappa}^{T}(x) \\
\kappa(x) & i \pi^{\prime}(x)
\end{array}\right)+\frac{i \varphi(x)}{\sqrt{\left(N_{f}+N_{v}\right) N_{v} N_{f}}}\left(\begin{array}{cc}
N_{v} \mathbb{1}_{N_{f}+N_{v}} & 0 \\
0 & \left(N_{f}+N_{v}\right) \mathbb{1}_{N_{v}}
\end{array}\right),
$$

where $\kappa(x)$ and $\bar{\kappa}(x)$ are independent $N_{v} \times\left(N_{f}+N_{v}\right)$ matrices with elements in the Grassmann algebra, $\pi(x)=\pi(x)^{\dagger}$ and $\pi^{\prime}(x)=\pi^{\prime}(x)^{\dagger}$ are traceless Hermitian matrices of dimension $N_{f}+N_{v}$ and $N_{v}$, respectively, $\varphi(x) \in \mathbb{R}$, and $\mathbb{1}_{n}$ is the $n$-dimensional identity matrix. Note that in the fully quenched case of $N_{f}=0$ also the singlet field needs to be included in the effective theory [9]. In this contribution, however, we restrict the discussion to the partially quenched case. The Lagrangian of the effective theory to leading order in $U(x), \partial_{\rho} U(x)$, and the quark mass matrix $M$ is given by

$$
\mathscr{L}=\frac{F^{2}}{4} \operatorname{Str}\left[\partial_{\rho} U(x)^{-1} \partial_{\rho} U(x)\right]-\frac{\Sigma}{2} \operatorname{Str}\left[M^{\dagger} U(x)+U(x)^{-1} M\right]
$$

with $M=\operatorname{diag}\left(m_{1}, \ldots, m_{N_{f}}, m_{v 1}, \ldots, m_{v N_{v}}, m_{v 1}^{\prime}, \ldots, m_{v N_{v}}^{\prime}\right)$ and low-energy constants $\Sigma$ and $F$. The Lagrangian for nonzero imaginary chemical potential is obtained by replacing the derivative $\partial_{\rho}$ with the covariant derivative $\nabla_{\rho}$ defined by

$$
\nabla_{\rho} U(x)=\partial_{\rho} U(x)-i \delta_{\rho 0}[C, U(x)]
$$

where $C=\operatorname{diag}\left(\mu_{1}, \ldots, \mu_{N_{f}}, \mu_{v 1}, \ldots, \mu_{v N_{v}}, \mu_{v 1}^{\prime}, \ldots, \mu_{v N_{v}}^{\prime}\right)$ and $i \mu_{i}$ is the imaginary chemical potential of quark flavor $i$. The supertrace $\operatorname{Str}$ is a generalization of the ordinary trace that satisfies $\operatorname{Str} A B=$ $\operatorname{Str} B A[6]$.

\section{Finite-volume corrections to $\Sigma$ and $F$}

In this section we consider the theory in a box of volume $V=L_{0} L_{1} L_{2} L_{3}$ in the Euclidean formalism. The temporal extent of the box is given by $L_{0}$. We use the $\varepsilon$-regime power counting [12] defined by

$$
V \sim \varepsilon^{-4}, \quad M \sim \varepsilon^{4}, \quad \mu \sim \varepsilon^{2}, \quad \partial_{\rho} \sim \varepsilon, \quad \xi(x) \sim \varepsilon .
$$

Note that the expansion in $\varepsilon^{2}$ amounts to an expansion in $1 / \sqrt{V}$. To leading order in this power counting the fields $\xi$ are effectively massless. Therefore, in order to obtain finite propagators, we separate the constant mode $U_{0}$ by the ansatz

$$
U(x)=U_{0} \exp \left[\frac{i \sqrt{2}}{F} \xi(x)\right]
$$


with $\int d^{4} x \xi(x)=0$. The leading-order Lagrangian is given by

$$
\mathscr{L}_{0}=\frac{1}{2} \operatorname{Str}\left[\left(\partial_{\rho} \xi(x)\right)\left(\partial_{\rho} \xi(x)\right)\right]-\frac{\Sigma}{2} \operatorname{Str}\left[M^{\dagger} U_{0}+U_{0}^{-1} M\right]-\frac{F^{2}}{4} \operatorname{Str}\left[\left[C, U_{0}^{-1}\right]\left[C, U_{0}\right]\right] .
$$

A careful analysis of the propagator, see Ref. [11], yields

$$
\left\langle\xi(x)_{a b} \xi(y)_{c d}\right\rangle_{0}=\bar{\Delta}(x-y)\left[\delta_{a d} \delta_{b c}(-1)^{\varepsilon_{b}}-\frac{1}{N_{f}} \delta_{a b} \delta_{c d}\right],
$$

where

$$
\langle\mathscr{O}[\xi]\rangle_{0}=\frac{\int d[\xi] \mathscr{O}[\xi] e^{-\int d^{4} x \mathscr{L}_{0}}}{\int d[\xi] e^{-\int d^{4} x \mathscr{L}_{0}}}
$$

$\bar{\Delta}(x)$ is the massless propagator without zero modes [13], and

$$
\varepsilon_{b}= \begin{cases}0 & \text { for } 1 \leq b \leq N_{f}+N_{v}, \\ 1 & \text { for } N_{f}+N_{v}<b \leq N_{f}+2 N_{v} .\end{cases}
$$

Note that the propagator does not depend on the valence quark number $N_{v}$. The propagator $\bar{\Delta}(x)$ is finite in dimensional regularization and contains the dependence on the volume. The contributions to the Lagrangian at next-to-leading order in $\varepsilon$ are given by

$$
\mathscr{L}_{2}=\mathscr{L}_{2}^{M}+\mathscr{L}_{2}^{C}+\mathscr{L}_{2}^{N}
$$

with

$$
\begin{aligned}
\mathscr{L}_{2}^{M} & =\frac{\Sigma}{2 F^{2}} \operatorname{Str}\left[M^{\dagger} U_{0} \xi(x)^{2}+\xi(x)^{2} U_{0}^{-1} M\right], \\
\mathscr{L}_{2}^{C} & =-\frac{1}{2} \operatorname{Str} U_{0}^{-1} C U_{0}[\xi(x),[C, \xi(x)]]-\frac{i}{2} \operatorname{Str}\left(U_{0}^{-1} C U_{0}+C\right)\left[\xi(x), \partial_{0} \xi(x)\right], \\
\mathscr{L}_{2}^{N} & =\frac{1}{12 F^{2}} \operatorname{Str}\left[\partial_{\rho} \xi(x), \xi(x)\right]\left[\partial_{\rho} \xi(x), \xi(x)\right]-\frac{1}{3 \sqrt{2} F} \operatorname{Str} U_{0}^{-1}\left[C, U_{0}\right]\left[\xi(x),\left[\partial_{0} \xi(x), \xi(x)\right]\right] .
\end{aligned}
$$

We will integrate out the fluctuations in $\xi$ in order to obtain an effective finite-volume partition function. The term $\mathscr{L}_{2}^{M}$ couples to $U_{0}$ and $M$ and corrects the leading-order mass term to

$$
-\frac{\Sigma}{2}\left[1-\frac{N_{f}^{2}-1}{N_{f} F^{2}} \bar{\Delta}(0)\right] \operatorname{Str}\left[M^{\dagger} U_{0}+U_{0}^{-1} M\right],
$$

where $\bar{\Delta}(0)$ in dimensional regularization is given by [13]

$$
\bar{\Delta}(0)=-\frac{\beta_{1}}{\sqrt{V}} .
$$

The so-called shape coefficient $\beta_{1}$ only depends on the quantities $l_{i}=L_{i} / V^{1 / 4}$ with $i=0,1,2,3$. The terms in $\mathscr{L}_{2}^{C}$ couple to $U_{0}$ and $C$ and correct the leading-order chemical potential term to

$$
-\frac{F^{2}}{4}\left[1-\frac{2 N_{f}}{F^{2}}\left(\bar{\Delta}(0)-\int d^{4} x\left(\partial_{0} \bar{\Delta}(x)\right)^{2}\right)\right] \operatorname{Str}\left[\left[C, U_{0}^{-1}\right]\left[C, U_{0}\right]\right]
$$


with

$$
\int d^{4} x\left(\partial_{0} \bar{\Delta}(x)\right)^{2}=-\frac{1}{2 \sqrt{V}}\left[\beta_{1}-\frac{L_{0}^{2}}{\sqrt{V}} k_{00}\right]
$$

where $k_{00}$ is another shape coefficient [14] that only depends on $l_{0}, \ldots, l_{3}$. Thus we can read off effective low-energy constants $\Sigma_{\text {eff }}$ and $F_{\text {eff }}$,

$$
\begin{aligned}
& \frac{\Sigma_{\text {eff }}}{\Sigma}=1-\frac{N_{f}^{2}-1}{N_{f} F^{2}} \bar{\Delta}(0), \\
& \frac{F_{\text {eff }}}{F}=1-\frac{N_{f}}{F^{2}}\left(\bar{\Delta}(0)-\int d^{4} x\left(\partial_{0} \bar{\Delta}(x)\right)^{2}\right) .
\end{aligned}
$$

This is the same result as previously derived for the unquenched theory [3, 4]. The terms in $\mathscr{L}_{2}^{N}$ and also potential contributions from the integration measure are not relevant for finite-volume corrections to $\Sigma$ and $F$ at next-to-leading order in $\varepsilon$, see Ref. [11] for details.

\section{The constant-mode integral}

In the infinite-volume limit the fluctuations in $\xi$ are suppressed and the theory becomes zerodimensional. It is therefore described by RMT, and the integral over the constant mode $U_{0}$ defined in Eq. (3.2) should recover the RMT result. A proof for the case of vanishing chemical potential is given in Ref. [11]. We also note that fixing topology to a single topological sector $v$ merely amounts to a change of the integration manifold of $U_{0}$ and therefore does not alter the discussion of the finite-volume corrections given above. For a detailed discussion of the theory in a single topological sector we refer to Ref. [11].

\section{Conclusions}

In this work we have calculated the partially quenched partition function of QCD at next-toleading order in the $\varepsilon$-expansion at nonzero imaginary chemical potential. We considered a theory with $N_{f}+N_{v}$ fermionic quarks and $N_{v}$ bosonic quarks, as formulated by Sharpe and Shoresh [9], in a finite volume $V$ with microscopic quark masses $M$, i.e., $M V \Sigma=\mathscr{O}\left(\varepsilon^{0}\right)$. The knowledge of the analytic form of the partially quenched partition function suffices to obtain all spectral correlation functions of the Dirac operator $\not D$. In this sense our results for the finite-volume behavior of the theory hold universally for all observables that can be obtained from spectral correlation functions of $\not D$. We found that the partially quenched partition function has the same finite-volume corrections as the unquenched partition function of QCD with $N_{f}$ quarks.

In Fig. 1 we show the finite-volume corrections at NLO to the low-energy constants $\Sigma$ and $F$ as a function of the box size $L$ in a symmetric box. Note that the effects of the finite volume increase with the number of sea quark flavors $N_{f}$ and that, depending on $N_{f}$, a box size of $3-5$ $\mathrm{fm}$ is necessary to reduce the effects of the finite volume at NLO to about $10 \%$. The effects are calculated at $F=90 \mathrm{MeV}$. In Fig. 2 we show the effect of an asymmetric box with $N_{f}=2$ and $L=2 \mathrm{fm}$. An important message of this figure is that the magnitude of the finite-volume corrections can be significantly reduced by choosing one large spatial dimension instead of a large temporal 

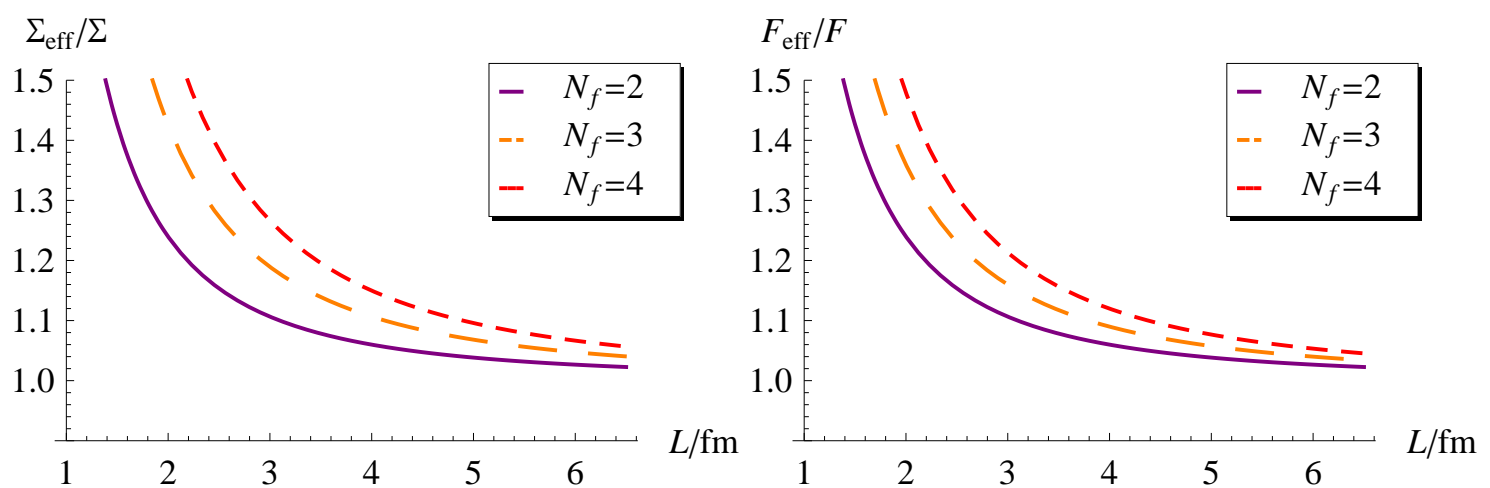

Figure 1: Volume dependence at NLO of the low-energy constants $\Sigma_{\text {eff }}$ (left) and $F_{\text {eff }}$ (right) in a symmetric box with dimensions $L_{0}=L_{1}=L_{2}=L_{3}=L$ at $F=90 \mathrm{MeV}$.
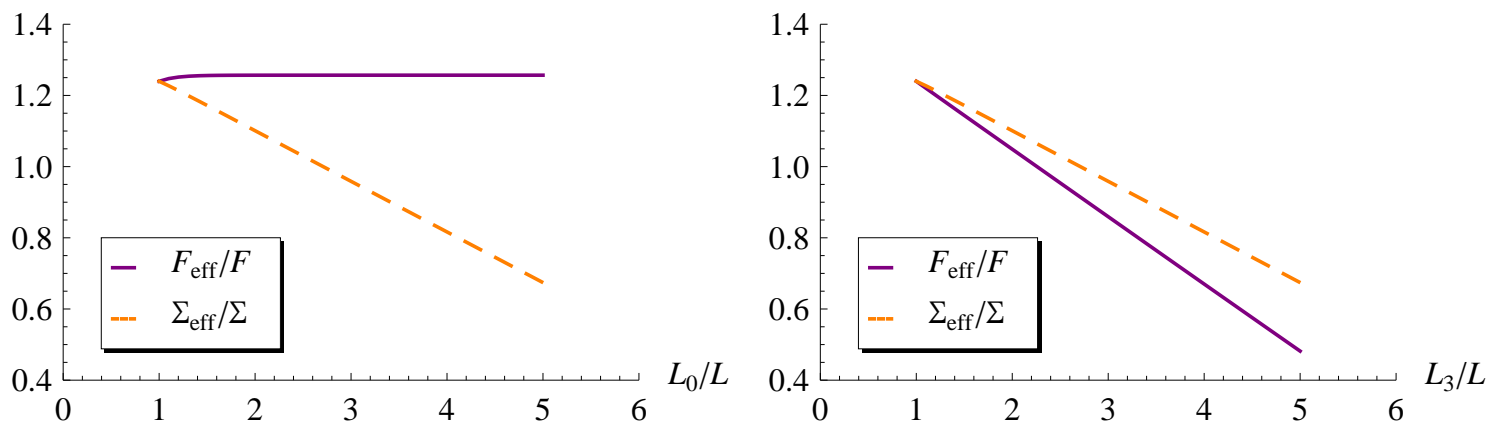

Figure 2: Effect of an asymmetric box with parameters $N_{f}=2, L=2 \mathrm{fm}$, and $F=90 \mathrm{MeV}$. We compare a large temporal dimension $L_{0}$ with $L_{1}=L_{2}=L_{3}=L$ (left) to a large spatial dimension $L_{3}$ with $L_{0}=L_{1}=$ $L_{2}=L$ (right).

dimension. The reason for this behavior is that the chemical potential only affects the temporal direction, see Eq. (2.5), and therefore breaks the permutation symmetry of the four dimensions. This manifests itself in the propagator

$$
\int d^{4} x\left(\partial_{0} \bar{\Delta}(x)\right)^{2}
$$

which, as shown in Eq. (3.14), contains a term proportional to $L_{0}^{2} / \sqrt{V}$, where $L_{0}$ is the size of the temporal dimension. This term leads to an enhancement of the corrections in case of a large temporal dimension. Choosing instead one large spatial dimension, the finite-volume corrections are reduced, unless the asymmetry is too large. For the parameters used in Fig. 2, the optimal value is $L_{3} / L \approx 2$.

This is good news. Many lattice simulations (at zero chemical potential) are performed with $L_{1}=L_{2}=L_{3}=L$ and $L_{0}=2 L$. To determine $F$, it suffices to introduce the imaginary chemical potential in the valence sector. Therefore, one can take a suitable set of existing dynamical configurations and redefine $L_{0} \leftrightarrow L_{3}$ before adding the chemical potential. ${ }^{1}$ This will minimize the finite-volume corrections for both $\Sigma$ and $F$, at least for the parameter values chosen in Fig. 2.

\footnotetext{
${ }^{1}$ Note that this procedure increases the temperature of the system by a factor of two. One needs to check that the system does not end up in the chirally restored phase, in which our results no longer apply.
} 


\section{Acknowledgments}

We thank Hidenori Fukaya and Shoji Hashimoto for stimulating discussions and the Theory Group of the INPS at KEK Tsukuba for their hospitality. This work was supported in part by BayEFG (CL) and by DFG and KEK (TW).

\section{References}

[1] P. H. Damgaard, U. M. Heller, K. Splittorff, and B. Svetitsky, A new method for determining F(pi) on the lattice, Phys. Rev. D72 (2005) 091501, [hep-lat/ 0508029 ].

[2] G. Akemann, P. H. Damgaard, J. C. Osborn, and K. Splittorff, A new chiral two-matrix theory for Dirac spectra with imaginary chemical potential, Nucl. Phys. B766 (2007) 34-67, [hep-th/0609059].

[3] P. H. Damgaard, T. DeGrand, and H. Fukaya, Finite-volume correction to the pion decay constant in the epsilon-regime, JHEP 12 (2007) 060, [arXiv: 0711 . 0167].

[4] G. Akemann, F. Basile, and L. Lellouch, Finite size scaling of meson propagators with isospin chemical potential, JHEP 12 (2008) 069, [arXiv: 0804 . 3809].

[5] S. F. Edwards and P. W. Anderson, Theory of spin glasses, J. Phys. F5 (1975) 965-974.

[6] K. B. Efetov, Supersymmetry and theory of disordered metals, Advances in Physics 32 (1983) 53-127.

[7] A. Morel, Chiral logarithms in quenched QCD, J. Phys. (France) 48 (1987) 1111-1119.

[8] C. W. Bernard and M. F. L. Golterman, Partially quenched gauge theories and an application to staggered fermions, Phys. Rev. D49 (1994) 486-494, [hep-lat/ 9306005 ].

[9] S. R. Sharpe and N. Shoresh, Partially quenched chiral perturbation theory without $\varphi_{0}$, Phys. Rev. D64 (2001) 114510, [hep-lat/ 0108003$].$

[10] T. DeGrand and S. Schaefer, Parameters of the lowest order chiral Lagrangian from fermion eigenvalues, Phys. Rev. D76 (2007) 094509, [arXiv: 0708.1731 ].

[11] C. Lehner and T. Wettig, Partially quenched chiral perturbation theory in the epsilon regime at next-to-leading order, arXiv:0909.1489.

[12] J. Gasser and H. Leutwyler, Thermodynamics of chiral symmetry, Phys. Lett. B188 (1987) 477.

[13] P. Hasenfratz and H. Leutwyler, Goldstone boson related finite size effects in field theory and critical phenomena with $O(N)$ symmetry, Nucl. Phys. $B 343$ (1990) 241-284.

[14] F. C. Hansen, Finite size effects in spontaneously broken $S U(N)$ x SU(N) theories, Nucl. Phys. $\mathbf{B 3 4 5}$ (1990) 685-708. 